\title{
Onomastic Remarks on Roman Sardinia
}

\author{
ROBERT J. ROWLAND, JR.
}

B

EFore the Romans seized and began occupying the island of Sardinia in 238 B. C., it had been settled by Punic-speaking Carthaginians and by indigenous natives (Protosardi), who had linguistic affinities with the indigenous populations of North Africa (Libyans), Spain (Iberians), and North-west Italy (Ligurians). ${ }^{1}$ Hence, the nomenclature of individuals from Roman Sardinia exhibits relationships with these areas, some of which have long since been pointed out.

First, the Punic names preserved primarily on Punic and neo-Punic inscriptions (fig. 1 and 2) as well as in Cicero and on Latin inscriptions (these comprise about 3 percent of the total): $:^{2}$

Aris (Cic. Scaur. frg. 2. 6), a notable from Nora;

belšlk (Punic, 32: Tharros), an architect;

bb'l (Neo-punic, 8: Bitia), a sufete;

bd' son of 'bd' (Punic, 26: Nora);

'bdh- son of t- (Punic, 32: Tharros), a sufete;

'bdkšr (Punic, 32: Tharros);

'bdmlqrt son of 'bdmlqrt (Neo-punic, 6: Sulci);

'bdšg- (Punic, 32: Tharros);

'bd'šmn son of himlk (Punic, 9: near S. Niccolò Gerrei), sufete;

Bostar (Cic. Scaur. frg. 2. 1), a notable from Nora;

Cn. Domitius Sincaius (Cic. Scaur. 43);

Himilco Idnibalis (hamlkt son of 'dnb'l) (CIL 10. 7513 = Neo-punic, 5: Sulci);

Himilco son of Himilco (hmlkt) (CIL 10. 7513 = Neo-punic, 5: Sulci);

ḩmlkt (Punic, 9: near S. Niccolò Gerrei), sufete;

ḥmy (Punic, 32: Tharros), sufete;

hanb'l (Punic, 34: Olbia);

Iulius M-sal (CIL 10. 7841: Vallermosa), sufete;

lḥn' of bodmlqrt, son of hạn' (Punic, 23. 2: S. Pietro);

mgn (Punic, 29: Nora);

mgn (Neo-punic, 6: Sulci), son of 'bdmlqrt;

1 See especially B. Terracini, "Gli studi linguistici sulla Sardegna preromana," Sardegna Romana, volume primo, Rome, 1936, 53-80; E. de Felice, "La Sardaigne dans la Méditerranée d'après la Toponymie des Côtes," Proceedings of the Eighth International Congress of Onomastic Sciences, The Hague, 1966, 147-173; V. Bertoldi, "Sardo-Punica," Parola del Passato 2 (1947) 3-38; G. Serra, "Appunti su l'elemento punico e libico nell'onomastica sarda," Vox Romanica 13 (1953/54) 51-65; G. Lilliu, review of G. Serra, Scritti vari di glottologia sarda, Studi Sardi 10-11 (1950-51) 579-594.

2 The texts of these inscriptions are those of G. G. Guzzo Amadasi, Le Iscrizioni fenicie e puniche delle colonie in Occidente, Studi Semiciti, 28, Rome, 1967, 83-136. 


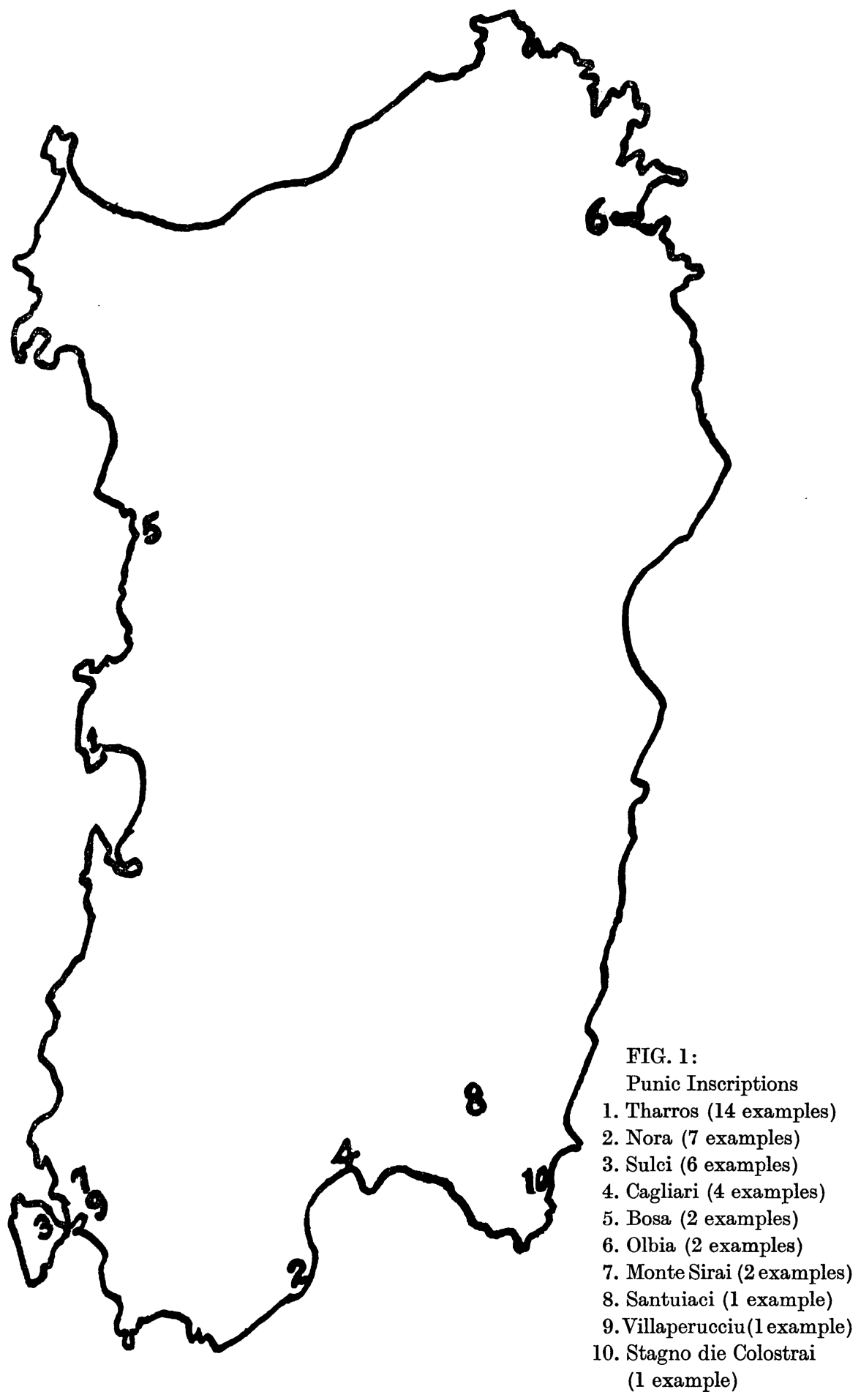


$84 \quad$ Robert J. Rowland, Jr.

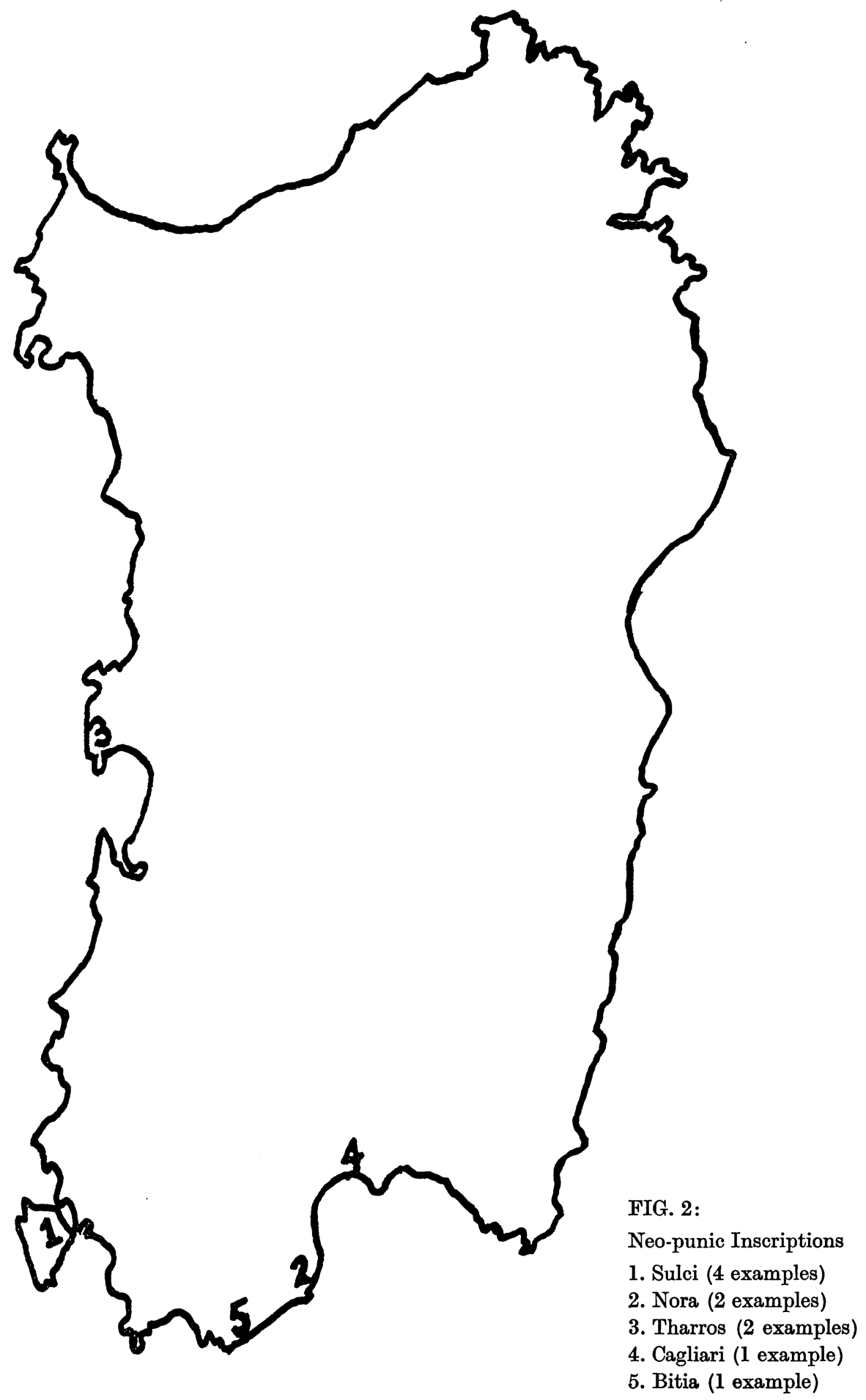


pdy son of 'rš (Punic, 27: Nora);

phly' (= Pullius ?) 'gbr (cf. Agbor: $C I L$ 8. 26701) son of mqr' (Neo-punic, 2: Sulci);

plks (= Felix) khrhșy (= Cressius ?: cf. CIL 10. 5247) (Neo-punic, 2: Sulci);

Phamea (Cic. Att. 13. 9; Fam. 7. 24. 2);

'rkrḥ (Neo-punic, 9: Tharros);

Saturninus 'nbrys (Neo-punic, 8: Bitia);

sp' (Neo-punic, 7 : Nora);

-.. son of 'tṣ (Punic, 23. 3: S. Pietro);

'zr (Punic, 32: Tharros);

-.-h (Neo-punic, 8: Bitia), sufete;

-.-hbrk son of șdq (Neo-punic, 1: Sulci);

-.-rt (Punic, 32: Tharros).

Further, many individuals bear nomina which are extremely common in Roman North Africa (about $4 \frac{1}{2}$ percent of the total):

Agria (S. 1. 247: Portotorres): common in Italy (IX: 8; X: 11; XIV: 11), but not in the North (V: $1 ; \mathrm{XI}: 1)$; especially common in Africa (VIII: 38$) ;^{3}$

Apertia (S. 1. 60: Cagliari): VIII: 8; a total of 6 in V, IX, XI, and XIV;

C. Apronius (S. 1. 25: Portotorres), Apronius Iuli- (S. 1. 74: Cagliari), C. Apronius C. f. Postumianus (S. 1. 251: Portotorres): VIII: 40; a total of 16 in V, X, XI, and XIV;

Bennia -ca Flavia -a Nereis O- (?) (S. 1. 55: Cagliari), L. Bennius L. f. Qui. Felix Minicianus (S. 1. 55: Cagliari), local notable: VIII: 18; a total of 14 in IX, X, and XI;

Marcus Caelestin(i ?)us (CIL 10. 3601: Misenum), sailor from Sardinia: VIII: 12; a total of 10 in II, X, XI, and XIV;

Cessia Sabina (CIL 10. 7586: Cagliari): VIII: 7; X: 1;

Favonia M. f. Vera (CIL 10. 7541 : Nora), from Cagliari, Q. Favonius A- (CIL 10. 7652: Cagliari), M. Favonius Callistus (CIL 10.7541: Nora), Augustalis, Favonius Donatus (CIL 8. 9202: Sûr Djuab), soldier in Cohors II Sardorum, Q. Favonius Ru- (CIL 10. 7652: Cagliari): VIII: 15; a total of 26 in $\mathrm{I}^{2}$; II; V, IX, X, XI, XII;

Gabinia (S. 1. 65: Cagliari), (St.) Gabinius (Bonu, 124-6), ${ }^{4}$ C. Gabinius Felix (S. 1. 65: Cagliari), P. Gabinius Faustus (CIL 10. 7645: Cagliari), Q. Gabinius A. f. Quir. Receptus (CIL 10. 7599: Cagliari), local notable: VIII: $60 ; \mathrm{X}: 32 ; \mathrm{XI}: 14 ; \mathrm{IX}: 10 ; \mathrm{XIV}: 6$; V: 1 ;

Gargilia L. f. Gemella (S. 1. 11: Sulci), Gargilius -ianus (CIL 10. 7656: Cagliari): VIII: 135; a total of 19 in IX, X, XI, XIV;

Gessia Allia ( $E E$ 8. 715: Cagliari): VIII: 11; V: 1; X: 4; XI, XII, XIV: 3 each;

s $\mathrm{S} .1$ = G. Sotgiu, Iscrizioni latine della Sardegna, Padova, 1961; S. $1969=$ G. Sotgiu, "Nuove iscrizioni inedite sarde," Annali delle Facoltà...dell'Università di Cagliari 32 (1969) 5-77; CIL = Corpus Inscriptionum Latinarum; EE = Ephemeris Epigraphica; Alföldy = G. Alföldy, Die Personennamen in der römischen Provinz Dalmatia, Beiträge zur Namenforschung, Beiheft 4, Heidelberg, 1969; Kajanto, Onomastic Studies = I. Kajanto, Onomastic Studies in the Early Christian Inscriptions of Rome and Carthage, Helsinki, 1963; Kajanto = I. Kajanto, The Latin Cognomina, Helsinki, 1965; Pape = W. Pape, Wörterbuch der griechischen Eigennamen, 3d ed., Braunschweig, 1884; Wuthnow = H. Wuthnow, Die semitischen Menschennamen in griechischen Inschriften und Papyri des Vorderen Orients, Leipzig, 1930. The formula IX: 8 (e. g.) means that there are eight examples of a name in $C I L$, volume 9. Names which are extraordinarily common and ubiquitous (Cassius, Petronius, Valerius, e. g.) have been left out of account in this study. Some names appear on more than one list.

4 Bonu $=$ R. Bonu, $E$ a dir di Sardegna, Cagliari, 1969, who provides, on pp. 105-168, a convenient list, with full references, of Sardinian saints (real and apocryphal). 
A. Helvidius (CIL 10. 7717: Cagliari): VIII: 7; XIV: 4; XI: 2; cf. Herennia M. f. Helvidia ( $E E$ 8. 718: Elmas);

M. Helvius Rogatus (CIL 10. 7597: Cagliari): VIII: 101; XIV: 18; XI: 27; V: 27;

Labinia P. f. ( $C I L$ 10. 7675: Cagliari), Labinius (CIL 10. 7675: Cagliari): VIII $+A E$, 1968, 632: 3; XII: 3;

Lerius Fronto (S. 1. 332: unknown location): VIII: 1 possible; XI: 1;

Luria Privata (S. 1. 265: Portotorres): VIII: 63; a total of 24 in IX, X, XI, and XIV;

Magneius L. f. Ouf. Sardus (CIL 10. 5663: Frusino), Magnia Lucretia ( $E E$ 8. 723 : near

Pirri), L. Magnius Fulvianus (CIL 10. 7946: Portotorres), tribunus militum, L. Magnius Fortunatianus Quirina (CIL 8. 3185: Lambaesis), from Cagliari; parallels with -ei- are rare (CIL 6. 28656: Magneia Donata), perhaps also $C I L$ 12. 4977: Manneia Primula; Magnius is more common: VIII: 88; a total of 23 in III, V, X, XI, XII, XIV;

Matius Resus (CIL 10. 7816: Pirri): VIII: 23; a total of 10 in IX, XI, XII, XIV;

Mevia Ur-.. (CIL 10. 7682: Cagliari): VIII: 46; a total of 35 in IX, X, XI, and XIV;

Q. Porcius Clarus (CIL 10. 8327: Portotorres), M. Porcius Felix (CIL 10. 7514: Sulci),

M. Porcius Impetratus (CIL 10. 7514: Sulci), local notable, M. Porcius M. 1. Primigenus (CIL 10. 7514: Sulci), magister Larum Augustalium: VIII: 43 ; a total of 32 in V, IX, X, $\mathrm{XI}$, and XIV;

Silius Tabernarius (S.1.268: Portotorres):VIII: 68;V:4;IX:8; X:19; XI: 14; XIV:21 ;

C. Sittius Egnatianus (S. 1. 273: Portotorres): extraordinarily common in VIII;

C. Tutius (CIL 10. 8320: Sulci): VIII: 8; X: 2; XI; XIV: 4 each.

As can be seen from fig. 3 , the distribution of these names closely parallels what we saw for the Punic names. There are also numerous other names (about 6 percent of the total) which are common in Roman North Africa, but which are so common elsewhere that they cannot be said to be peculiarly African (fig. 4):

Sex. Appuleius (CIL 10. 7627: Cagliari): V: 34; IX; 11; X: 83; XI: 11; XIV: 25; VIII: 24;

Aquilia Pompeia (CIL 10. 7628: Cagliari): VIII: 26; X: 25; V, XIV: 14 each; XI: 12; IX: 8;

Arria Chia (S. 1. 100: Cagliari), Arria Crispinilla (S. 1. 100: Cagliari), Arrius -corus (CIL 10. 7949: Portotorres), Arrius Callistus (S. 1. 100: Cagliari), Arrius Iscini- (CIL 10. 7917 : Cornus), C. Arrius Laetus (CIL 10. 7591 : Cagliari), miles cohortis Sardorum, Arrius Saturninus (CIL 10. 7964: Portotorres): X: 84; VIII: 44; IX: 28; XI: 49; XIV: 24;

Aruntius (CIL 10.7867 : Fordongianus) : VIII: 71 : XI: $27 ; \mathrm{X}: 28 ; \mathrm{IX}: 16 ; \mathrm{XIV}: 14 ; \mathrm{V}: 12$;

Caesonia Soteris (CIL 10. 7635: Cagliari): V: 4; VIII: 11 ; IX: 1 ; X: 19; XI: 5; XIV : 8;

Cornificia Gemina (CIL 10. 7645: Cagliari): X: 13; VIII: 7; XIV: 5; XI: 1 ;

Egnatia (CIL 10. 7813: Pirri), Egnatia (CIL 10. 7825: Sestu), Egnatia C. f. Iunia ( $E E$ 8. 717: Cagliari): XI: 58; VIII: 50; X: 45; IX: 35; V: 21; XIV: 12;

Fabricia Primiginia (CIL 10. 7593: Cagliari), L. Fabricius Faustus (CIL 10. 7845: Usellus), local notable, C. Fabricius Ianuarius (CIL 10. 7593: Cagliari), archigybernus: VIII: $38 ; \mathrm{X}: 20$; XI: 11 ; XIV: $10 ; \mathrm{V}: 9$;

Lollia Philonice (CIL 10. 7963: Portotorres), C. Lollius Crescentianus (CIL 10. 7670: Cagliari): VIII: 69 ; X: 48; IX: 39; XIV: $21 ; \mathrm{XI}: 17$;

Lucretia Fortunata (CIL 10. 7680: Cagliari), Lucretia Piaeris (CIL 10. 7680: Cagliari), Lucretia Venusta (CIL 10. 7964: Portotorres), Lucretius (CIL 10. 7584: Cagliari), L. Lucretius (S. 1. 219: Austis), Lucretius -.. Felix (CIL 10. 7679: Cagliari), Lucretius Fortunatus (CIL 10. 7680: Cagliari), L. Lucretius L. 1. Pacatus (CIL 10. 7887 : Austis), Lucretius Secundus (CIL 10. 7887: Austis), collibertus of preceding: X: 41 ; VIII: 38; XI: 21 ; IX: 19 ; XIV: 19; 


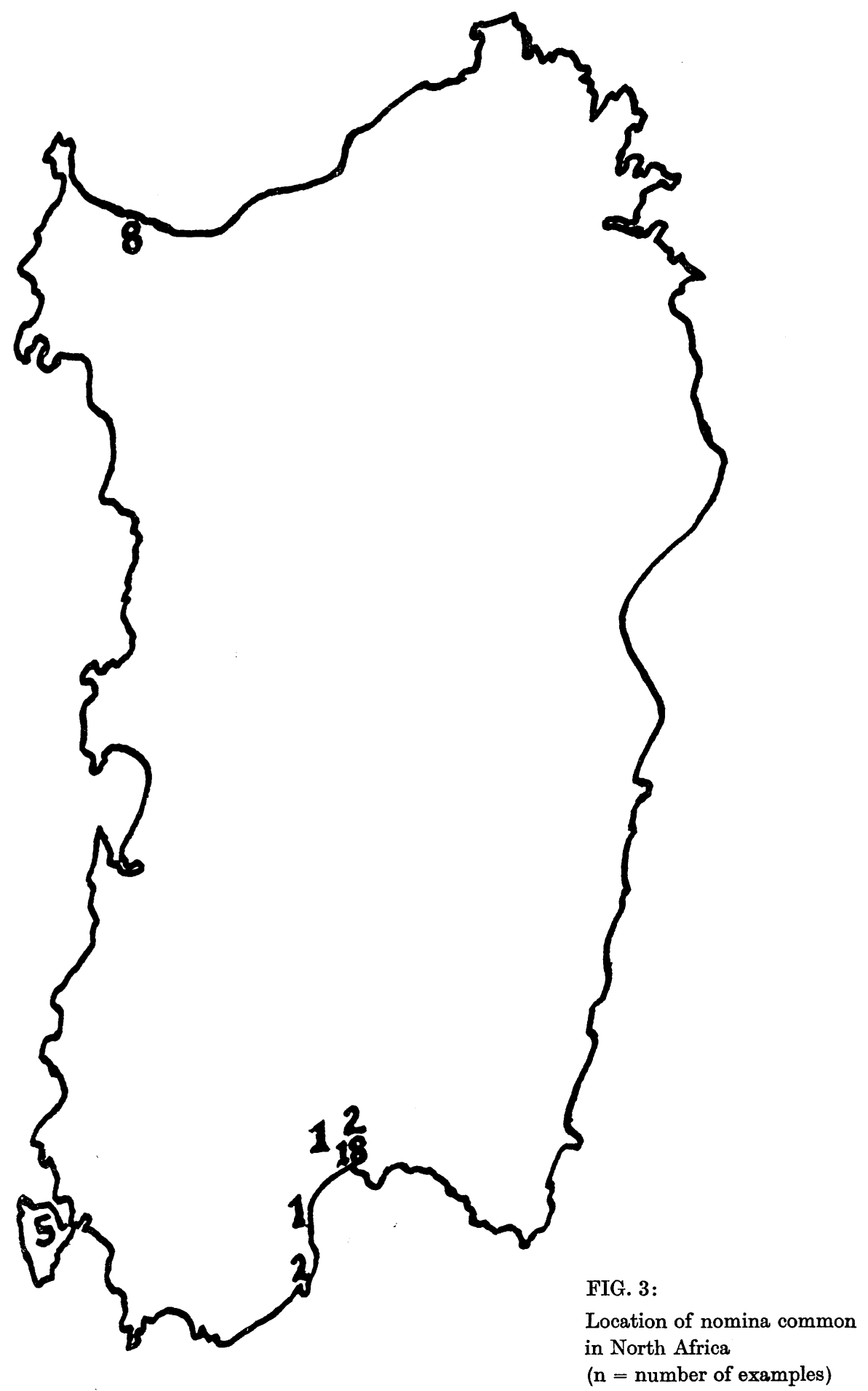


$88 \quad$ Robert J. Rowland, Jr.

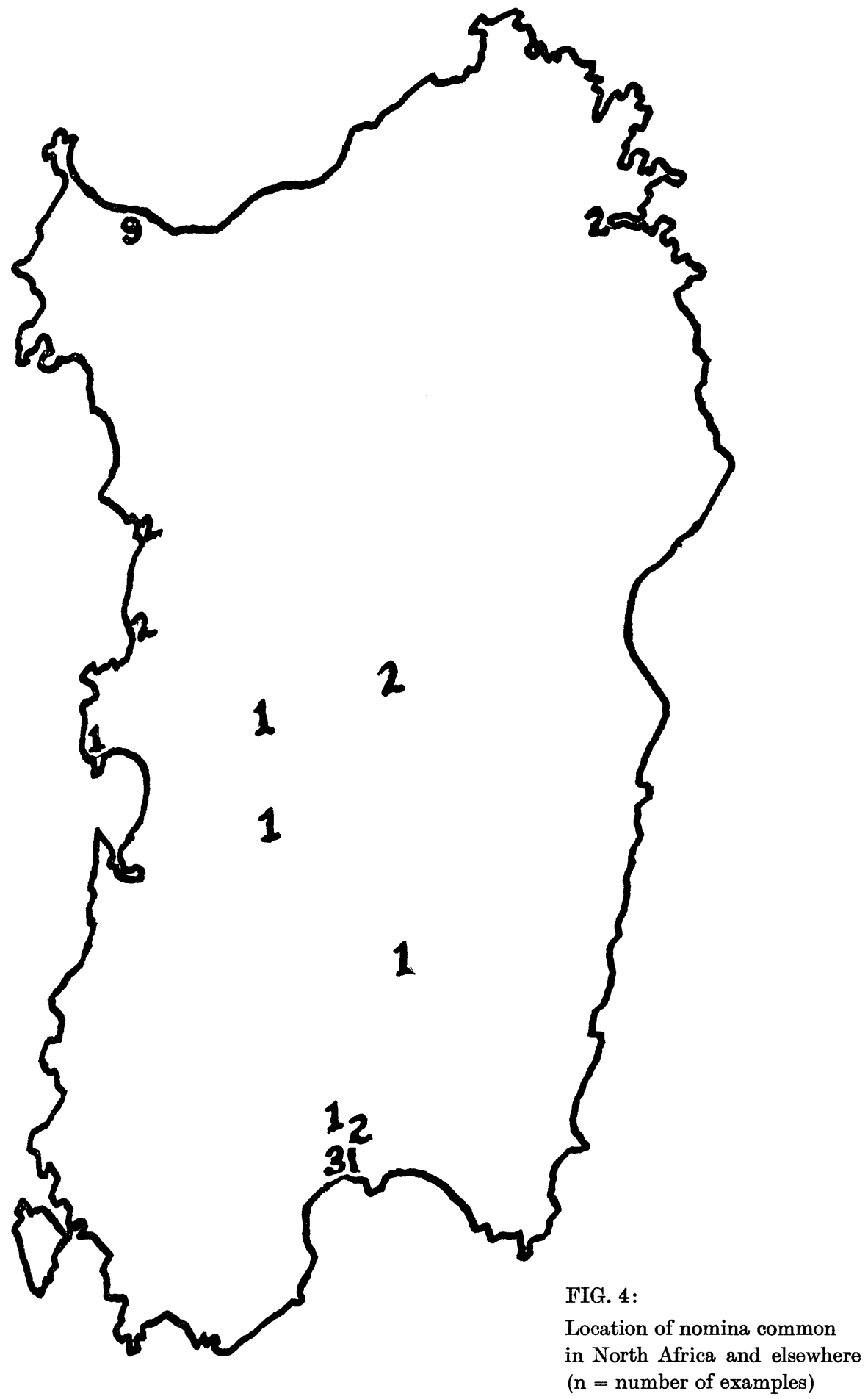


Messia (CIL 10. 7655: Cagliari): VIII: 31 ; X: 26; XI: 22; IX: 16;

M. Nonius Aquilinus (CIL 10. 3598: Misenum): X, VIII: 89 each; IX: 48;

C. Oclatius Macer, Caralitanus (CIL 10. 7891): V: 6; VIII: 4; X: 2; XI: 4; XIV: 2;

Otacilia Itageni (S. 1. 269: Portotorres): X: 14; VIII: 17; IX: 1; XI: 7; XIV: 23;

M. Papirius Sp.f. (CIL 10. 7688: Cagliari): V: 38; VIII: 37; XI: 36; X: 14; IX: 13; XIV: 4;

Rutilia Amalia (S. 1. 234: Bosa), Rutilia C.f. Ametghesa (CIL 10. 7699: Cagliari), Rutilia Xanthippe (CIL 10.7817: Pirri), Q. Rutilius (CIL 10.7939: Bosa), Rutilius Ostesis (S. 1. 272: Portotorres), Rutilius Veratianus (CIL 10. 7817: Portotorres): VIII: 38; IX: 35; X: 31; XI: 24; V: 20; XIV: 30;

Sei- (Seia ?) (CIL 10. 7987: Olbia), Seia Ursa (CIL 10. 7657: Cagliari): VIII: 57; V: 15; XI: $24 ; \mathrm{X}: 23$; IX: 4 ;

Sergia Melitine (CIL 10. 7701 : Cagliari), liberta, Sergis (CIL 10. 7775: Cagliari), Sergius Antoninus (CIL 10. 7707: Cagliari), Q. Sergius Q. f. Quir. Quadratus (CIL 10. 7915: Cornus), local notable: VIII: 36 ; X, XI: 22 each; IX: 15; V: 10;

M. Servil- (CIL 10. 7987: Olbia), Servilia C. l. Mo- (S. 1. 251 : Portotorres), Publius Serbilius (CIL 10. 7628: Cagliari): VIII: 71; V: 41; X: 34; IX: 22; XI: 17;

Sextia Prima (CIL 8. 9198: Sûr Djuab), wife of Abillahas Rummeus, soldier in Cohors II Sardorum: V: 34; VIII: 47; IX: 16; X: 26;

Sossius (S. 1. 227: Tharros) VIII: 33; X: 12; XI: 26; XIV: 12; IX: 5;

Stertinia Fortunata (CIL 10. 7703: Cagliari), Stertinius Abitus (CIL 10. 7704: Cagliari), C. Stertinius Aelianus (CIL 10. 7703: Cagliari), C. Stertinius Bacchyllas (CIL 10. 7703: Cagliari), C. Stertinius Felix ( $C I L$ 10. 7857 : Donigala): V: 2; VIII: 8; IX: 1; X: 13; XI: 5; XIV: 5 ;

Tatia Inc- (CIL 10. 7689: Cagliari): X: 18; VIII: 7; IX: 5; XIV: 2; XI: 3; V: 1;

Verati- (S. 1. 121: Cagliari), Veratius Hermeros (CIL 10. 7956: Portotorres): XI: 4; VIII, XIV: 6 each;

T. Vinius Beryllus, father and son (CIL 10. 7719: Cagliari): X: 12; VIII: 9; IX: 7; $\mathrm{XI}: 3$; XIV: 1.

Further, of African extraction will have been some of the bearers of such ubiquitous names as Cornelius, Cassius, Valerius, not to mention the imperial names Iulius, Claudius, Flavius, Aelius, Ulpius. For these, cognomina can assist, for certain cognomina were particularly favored in Punic speaking lands. ${ }^{5}$ The following list encompasses those cognomina which are most common in Roman Africa and are attested in Sardinia (fig. 5); they comprise about 16 percent of the total:

Abeddea (= Habetdeus) (CIL 10. 7744: Cagliari); cf. Kajanto, 217;

Acrabas (2 examples); Kajanto, 306;

Adeodata (CIL 10. 7745: Cagliari); Kajanto, 216;

Admonius (= Ammonius) (Concilia Galliae [314-506] 1. 15. 70): Presbyter de civitate Caralis; VIII: 3 ; IX: 2 ; X: 1 ; V: 1 ; XIV: 1 ; observe C. Ammonius Montanus, born at Alexandrea $(C I L$ 6. 3093) and C. Hammonius Fortis, born in Egypt (CIL 10. 3381);

5 Cf. H. G. Pflaum, "Remarques sur l'onomastique de Castellum Celtinanum," Carnuntiana (ed. S. Swoboda), Graz, 1956, 126-151; L. A. Thompson, "Settler and Native in the urban Centres of Roman Africa," Africa in Classical Antiquity (ed. L. A. Thompson and J. Ferguson), Ibadan, 1969, 132-181 ; J. Marion, "La Population de Volubilis à l'époque romaine," Bulletin d'archéologie marocaine 4 (1960) 133-187. 


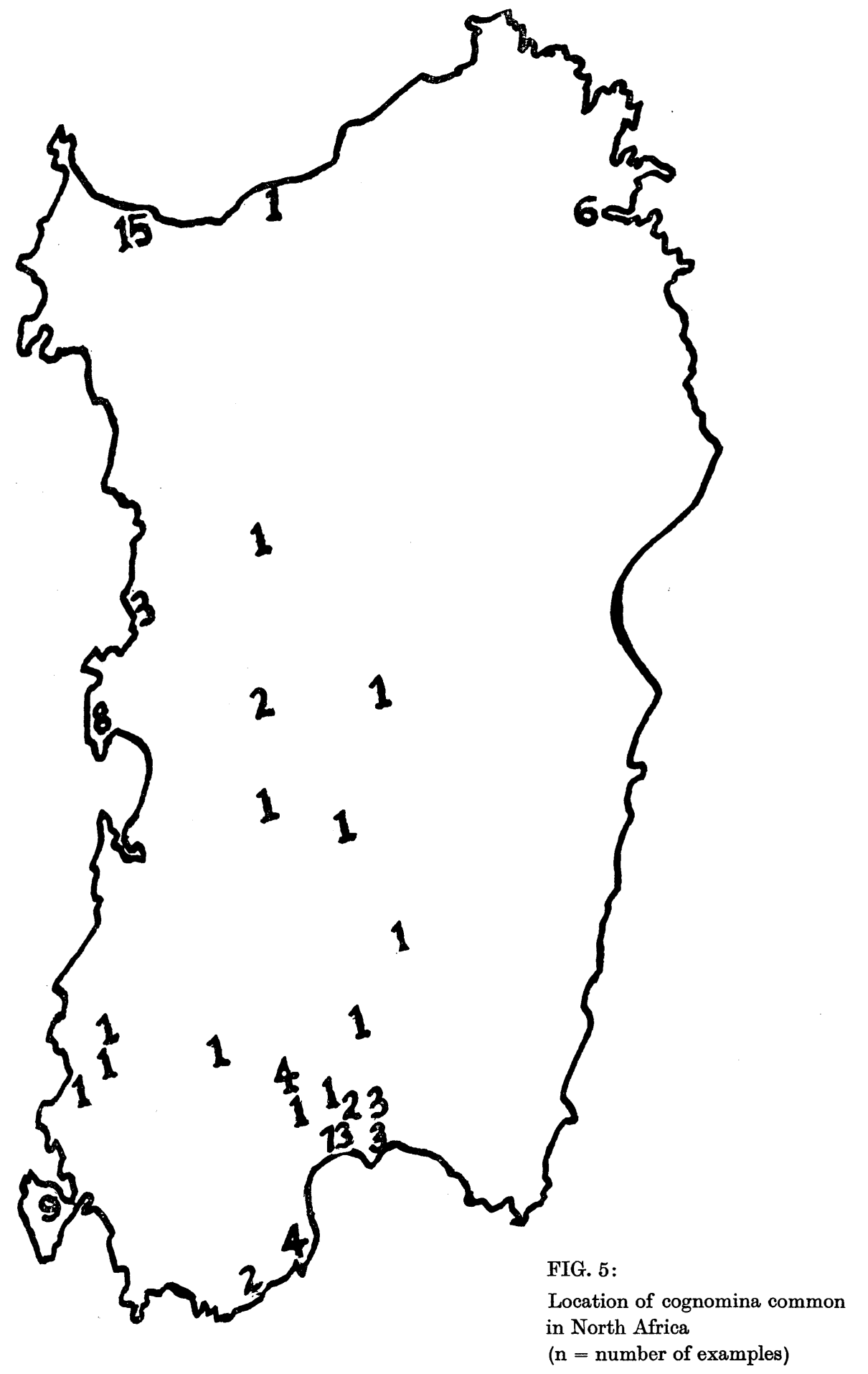


Ampelius (Symm. Epis. 2. 33A): VIII: 5; 1 each in V, IX, XI;

Asellus (2 examples); Kajanto, 325;

Asinarius (CIL 10. 7868: Fordongianus); the only other example is from Carthage $(C I L$

8. 13468);

Aufidianus (CIL 10. 7676: Cagliari); Kajanto, 141 ;

Benenatus (S. 1. 329: Olbia); VIII: 22; V: 2; XII: 2;

Bernaceus $(C I L$ 10. 7752: Cagliari) $=$ Bernaclus: $C I L$ 8. 2292, 13992-3, 23053;

Bonifatius (2 examples); VIII: 59; V, IX, XI: 1 each;

Bonus Homo (CIL 10. 7783: Cagliari); Kajanto 274;

Calvisius/-anus (2 examples); Kajanto, 143;

Castus (2 examples); Kajanto, 251;

Catella (Greg. Ep. 1. 60, 62); VIII: 3;

Celsus (S. 1. 307: Castelsardo); Kajanto, 230;

Dativus (S. 1. 14: Sulci); Kajanto, 298;

Deusdedit (S. 1969, 88: Cagliari); Kajanto, 217;

Donatus (3 examples); Kajanto, 298;

Epiphanius (2 examples); VIII: 8;

Eutychius/-anus (2 examples); Kajanto, Onom. Stud., 74, 83;

Felix (29 examples); Kajanto, 272;

Fortunatus (13 examples); Kajanto, 273;

Fuscus (2 examples); VIII: 65; XI: 22; IX: 12;

Gaudiosa ( $A E, 1966,174$ : Portotorres); Kajanto, 260;

Germanus (5 examples); Kajanto, 201;

Hilarus (5 examples); Kajanto, 261; VIII: 59;

Honoratus (7 examples); Kajanto, 279;

Ianuarius (14 examples); Kajanto, 218;

Innocentius (Greg. $E p$. 13. 6); Kajanto, 252;

Iucundula (CIL 10. 7815: Pirri); Kajanto, 282;

Iustus (CIL 10. 7901: Tharros); VIII: 54;

Marinianus (Greg. Ep. 1. 59, 10. 3); VIII: 8;

Martialis (3 examples); Kajanto, 212;

Mustulus (S. 1. 14: Sulci); Kajanto, 17f.;

Peregrin- (S. 1. 124a: Cagliari); Kajanto, 313;

Perpetua (S. 1. 107, 108: Cagliari); Kajanto, 274;

Quadratus (3 examples); Kajanto, 232;

Quintasius (Conc. Gall. 1. 15. 70); Kajanto, 174;

Quobuldeus (CIL 10. 7769: Cagliari); Kajanto, 217;

Reparata (2 examples); Kajanto, 355;

Rogatus (9 examples); Kajanto, 297;

Salsula (CIL 10. 7643: Cagliari); Kajanto, 261 ;

Saturninus (16 examples); Kajanto, 213;

Saturus (S. 1. 338: unknown location); Kajanto, 233;

Tabernarius (S. 1. 268: Portotorres); Kajanto, 322;

Urbanus (9 examples); Kajanto, 311.

Obviously, not every individual who bore one of these names can be related to Africa; Trosius Felix (CIL 10. 7709: Cagliari), for example, is probably Venetic (Alföldy, 129). However, they do provide a further indication of the extent to which Roman Sardinia was indebted to its African background. 
There are still further names whose best referents seem to be Roman Africa :

Abillahas Rummeus ( $C I L$ 8. 9198). soldier in Cohors II Sardorum, is probably African (cf. Wuthnow, 9); he may have been recruited locally;

Aedilius (CIL 10. 7884-5: Austis); cf. $C I L$ 8. 15470 (Aedilius Felix);

Aelia Annia (S. 1. 308: S. Teresa di Gallura); may be Semitic: Wuthnow, 23;

Aelia Bonavia (S. 1. 98: Cagliari); may be African, for Bonavia seems to be limited to CIL 8 (2 examples);

Ammia (Notizie degli Scavi, 1913, 90: Grugua); may be Semitic (Alföldy, 147);

Barex (S. 1. 175: Nuragus); perhaps = Baric (VIII: 33);

Benets (S. 1. 177: Ales); perhaps = Benis (Wuthnow, 35) or Venetus;

Brumasius (Vita Fulgenti, 51); cf. CIL 8. 23020. 3;

Burce (CIL 10. 7809: Pirri); cf. CIL 8. 4897; Pape, 225;

Buttis (CIL 10. 7634: Cagliari); cf. $C I L$ 8. 27864, 10. 2481, and (perhaps) Pape, 226;

Celele (S. 1. 177: Ales); a Semitic name (Wuthnow, 64); cf. Preisigke, 170;

Karitus (CIL 10. 7876: Fordongianus); cf. $C I L$ 8. 1911, 8576;

Mislius (S. 1. 177: Ales); cf. EE 5. 330;

Netus (S. 1. 172: Gergei); may be related to Netunus (CIL 10. 7864, 8. 23250); but cf. Holder, 2. 738 (Netis);

Sabdaga (S. 1. 177: Ales) and Zabarda (Greg. Ep. 4. 25); probably Semitic (Wuthnow, 48); cf. the names Zaba, Zabbur, Zabda in CIL 8;

Taretius Faric Corneli C. (?) (S. 1. 207: Samugheo); cf. CIL 8. 9467: Faricus;

Tarsalia (S. 1. 182: Seulo); cf. CIL 8. 18085a. 16 (Tarsa);

Valeria Amoccada ( $C I L$ 10. 7842: Vallermosa) and ---mocada/ebenni Ver- (CIL 10. 7848: Usellus) seem to be African;

Valentius ( $E E$ 8. 735: Telti); cf. $C I L$ 8. 21624 (Valentia Ianuaria);

Verona (CIL 10. 7843: Vallermosa); cf. CIL 8. 20321 (Veromna);

---miccina (CIL 10. 7751: Cagliari), ---thrid (CIL 10. 7812: near Pirri), ---uodin stam -.. (S. 1969, 2: Nora), although fragmentary, seem African.

For the distribution of these names, see fig. 6. As could perhaps be expected, there are numerous individuals from Roman Sardinia who bear names whose best parallels are in central and southern Italy. Two individuals proclaim themselves to be Faliscans $:^{6}$

K. Latrius K. f. $\left(C I L 1^{2} .364 \mathrm{a}=11.3078,7483\right)$; cf. $A E$, 1954, 96a;

C. Salvena Voltai f. (ibid.).

There is a fairly large body of names, whose distribution is of some importance (fig. 7), and which has parallels in the regions covered by CIL 9 (Calabria, Apulia, Samnium, Sabini, Picenum), 10 (Bruttium, Lucania, Campania, Sicilia), 11 (Aemilia, Etruria, Umbria), and 14 (Latium); these comprise about 7 percent of the total:

C. Acilius Marcianus, Caralitanus (CIL 10.6574: near Vellitri): common in North Italy (to which we shall return below), but also elsewhere (V:23; IX: 15; XI: 19; XIV: 36);

Acareltorte (S. 1. 217: Ortueri), cf. $C I L$ 10. 8053.149 (Acar-: Oristano): apparently unique, but cf. Acercelinio (R. Hirata, L'Onomastica falisca [Firenze, 1967], p. 31);

- Cf. Monte Sirai II, Studi Semitici, 14, Rome, 1965, tavola XLIV ("Vaso di tipo falisco"). 
Onomastic Remarks on Roman Sardinia

93

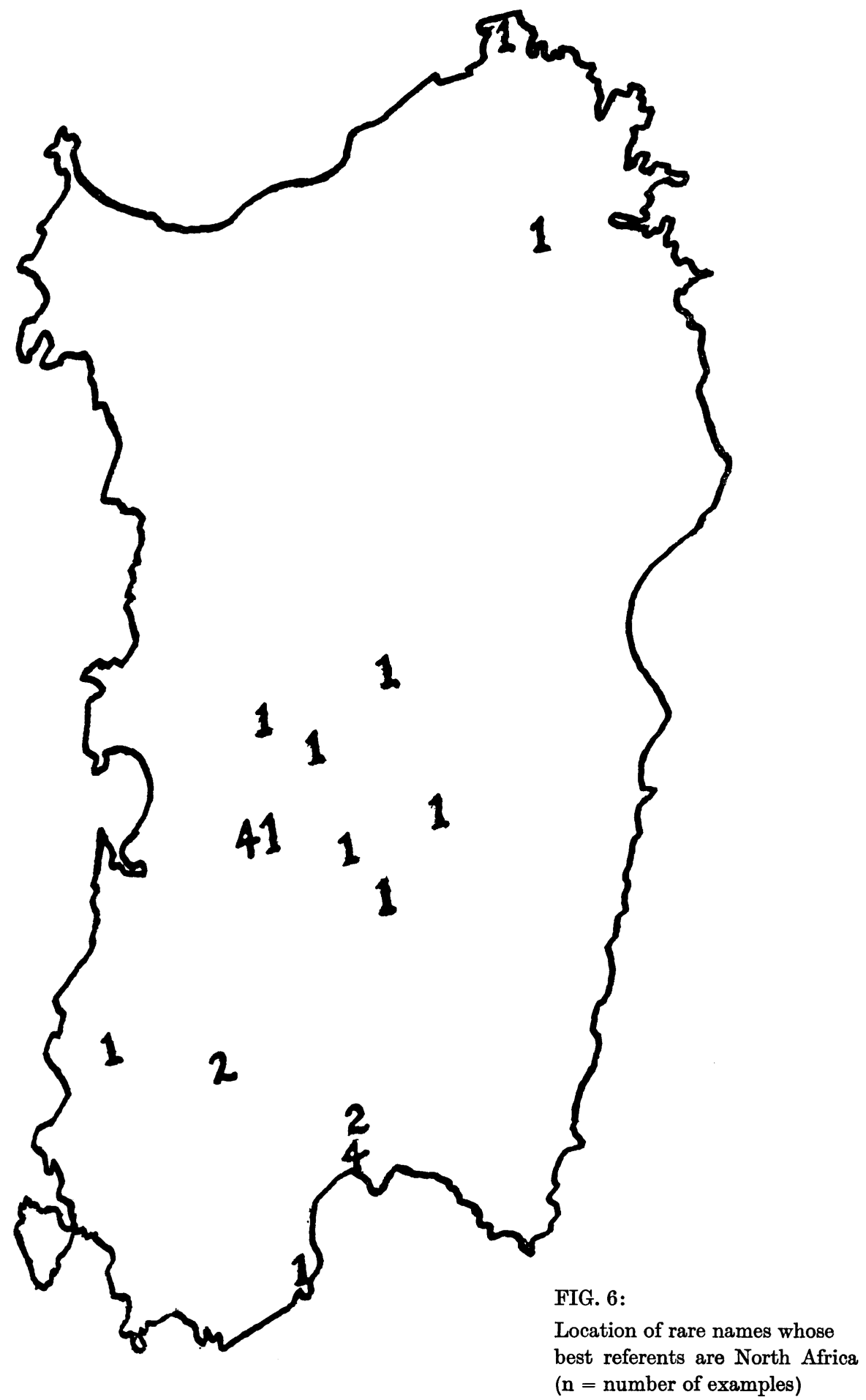


$94 \quad$ Robert J. Rowland, Jr.

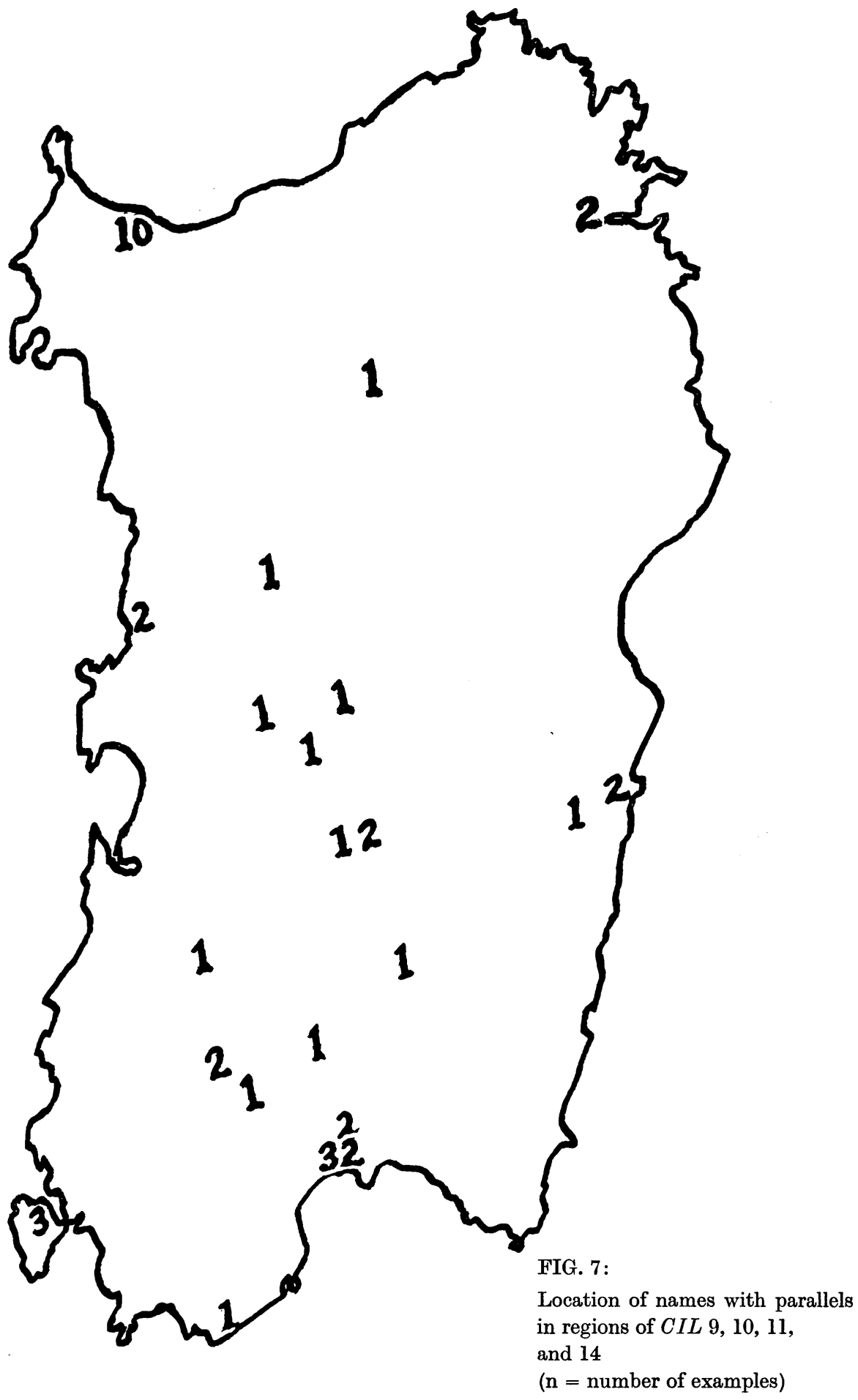


Aelius Tosuna (S. 1. 153: Pirri): apparently unique, but (perhaps) cf. Tosnos (CIL 11 . 2245: Clusium);

Agisinus (CIL 10. 7855: Tortoli): apparently unique, but (perhaps) cf. Agisenna (CIL 11. 3233: Nepet);

D. Alarius Pontificalis, Caralitanus (CIL 10. 7891): Pontificalis seems to be unique; Alarius is paralleled only by Alaria Saturnina ( $C I L 9.1299)$;

M. Alenius Tard- (CIL 10. 7722: Vallermosa): V: 6; IX: 3; XI: 8;

Allenia Urbana (CIL 10. 7839: near Vallermosa): X, XI: 1 each;

Sex. Appuleius (CIL 10. 7627: Cagliari): V: 34;IX:11; X: 83; XI:11; XIV: 25;VIII: 24;

Aquilia Pompeia (CIL 10. 7628: Cagliari): VIII: 26; X: 25; V, XIV: 14 each; XI: 12;

IX: 8;

Arria Chia (S. 1. 100: Cagliari), Arria Crispinilla (S. 1. 100: Cagliari), Arrius -corus (CIL 10. 7949: Portotorres), Arrius Callistus (S. 1. 100: Cagliari), Arrius Iscini- (CIL 10. 7917 : Cornus), C. Arrius Laetus, miles cohortis Sardorum (CIL 10. 7591 : Cagliari), Arrius Saturninus (CIL 10. 7964: Portotorres): X: 84; VIII: 44; IX: 28; XI: 49; XIV: 24;

Ateius Victorini (S. 1. 252: Portotorres): XI: 20; IX: 6; X: 13; XIV: 14; V: 4;

C. Avidius Speratus, father and son (S. 1. 178: Nurallo): IX: 29; X: 12; XI: 15; XIV: 5; V: 3; VIII: 1;

Aviena Philumena (CIL 10. 7521 : Sulci), Sex. Avienus Callicles (ibid.): XI: 5; X: 3; XIV, VI: 1 each;

Babullius -marus (CIL 10. 7633: Cagliari), Q. Babullius Urbanus (ibid.): X: 9; V: 4; IX: 1;

Caelonius (S. 1. 72: Cagliari): cf. CIL 9. 313: Caelonia Saturnina (unless = Caeionius for Ceionius);

Caesonia Soteris (CIL 10. 7635: Cagliari): V: 4; VIII: 11 ; IX: 1; X: 19; XI: 5; XIV: 8;

Calbisius Primitibus (CIL 10. 7636: Cagliari): V: 15; VIII: 12; IX: 3; X: 15; XI: 12; XIV: 3;

Capri(us ?) (S. 1. 89: Cagliari): X: 11; V: 3; IX: 8; XI: 1;

Cornificia Gemina (CIL 10. 7645: Cagliari): X: 13; VIII: 7; XIV: 5; XI: 1 ;

Costin(i ?)us (CIL 10. 7981: Olbia): cf. $C I L$ 11. 6695. 30 (Costini), 3. 12010. 12 and 12014. 215 (Costnius);

Critonius Hermes (CIL 10. 7921 : Cornus): X: 7; XIV: 6; IX: 4; V, XI: 3 each; VIII: 1 ; Cursus (or Cursius) ( $E E$ 8. 737: Olbia); cf. CIL 10.2141 (Cursus), ILG 228 (Cursi f.); Ducenius Faustus (CIL 10. 7716: Cagliari): X: 13; XI: 12; V: 3; XII, XIV: 1 each;

A. Egrilius A. f. Plarianus (CIL 10. 7955: Portotorres): XIV: 120; VIII: 27; Plarius/ Plarianus is more rare: Kajanto, 153 ; $C I L$ 11. 1075 add.; $A E, 1968,6 \mathrm{a} ; 1969,87$;

Enii (CIL 10. 7814: near Pirri), M. Hennius Simphorus (CIL 10. 7647 : Cagliari): V, IX: 38 each; X: 18; VIII: 12 ; XI: 8; XIV: 3 ;

Q. Fufius Celsus (CIL 10. 7948: Portotorres), Q. Fufius Proculus (ibid.): V: 3; VIII: 10 ; IX: $27 ; \mathrm{X}: 6 ; \mathrm{XI}: 8 ; \mathrm{XIV}: 1$;

C. Fusius Curadronis f. (CIL 10. 7854: Ilbone), miles classis: cf. CIL 2. 6064; 9. 6080, and 11. 6695. 45 (Fusi Scae, indexed as Fusius Scae..);

Gelasinus (S. 1. 66: Cagliari): IX: 1; X: 2; XIV: 3;

Messia (CIL 10. 7595: Cagliari): VIII: $31 ; \mathrm{X}: 26$; XI: 22; IX: 16;

Milonius (S. 1. 224: Ozieri): VI, XI: 3 each; 8. 5618; $A E$, 1930, 72; 1937, 62;

Novia Hygia (S. 1. 170: Nuraminis): X: 59; VIII: 19; IX: 18;

Quinta Numitoria (S. 1. 268: Portotorres), D. Numitorius Agisini f. Tarammo (CIL 10. 7855: Tortoli): V: 1 ; VI: 40; VIII: 5 ; IX: 5 ; X: 15; XI: 5; XIV: 8 ;

Otacilia Itageni (S. 1. 269: Portotorres): X: 14; VIII: 17; IX: 1; XI: 7; XIV: 23;

Paccia Ingenua (CIL 10. 7687: Cagliari), Paccius Coillius (ibid.), L. Paccius Cornelianus (S. 1. 270: Portotorres): X: 50; V: 7; VIII: 8; IX: 25; XI: 24; XIV: 1; 
L. Peducaeus Apollinaris (CIL 10. 7520: Sulci), C. Peducaeus Marcellus (CIL 10. 7838 : Decimopuzzu), M. Peducaeus Plautius (Neo-punic, 8: Bitia): X: 4; VIII: 2; V: 1; IX: 5; $\mathrm{XIV}: 4 ; \mathrm{XI}: 11$;

Q. Planius Sardus Eggius Ambibulus: cf. H-G. Pflaum, Bull. Arch. Com. Trav. Hist., 1963-1965, 143-51;

Ruffia Marcella C.f. (CIL 10. 7586: Cagliari): V: 6; VIII: 4; IX: 2; X: 6; XI: 19; XIV: 1 ;

Rusticelius Felix (CIL 10. 7597: Cagliari): V: 1; VI; 28; VIII: 1; X: 1; XI: 2; XIV: 6;

Silvanus Tantilianus (S. 1. 41 : S. Gavino): for Tantilius, cf. $C I L$ 6. 24714; 10. 1574, 2994 (2 examples), 7653;

Stertinia Fortunata (CIL 10. 7703: Cagliari), Stertinius Abitus (10. 7704: Cagliari), C. Stertinius Aelianus (10. 7703: Cagliari), C. Stertinius Bacchyllas (ibid.), C. Stertinius Felix (10. 7857: Donigala): V: 2; VIII: 8; IX: 1; X: 13; XI: 5; XIV: 5;

Sutoria Athenais (CIL 10. 7706: Cagliari), Sutoria Euporis (10. 7707: Cagliari), L. Sutorius Cornelianus (10. 7705: Cagliari), L. Sutorius Nicephorus (ibid.): V: 2; IX, XI: 1 each; VIII: $6 ; \mathrm{X}: 8$;

C. Tamudius Cassianus (CIL 10. 3636: Misenum), miles classis: VI: 4; IX: 3; XI: 2; VIII, $\mathrm{X}, \mathrm{I}^{2}$ : 1 each; $A E, 1951,217$;

Tarcuinus Fili f. Neroneius (S. 1. 209: Samugheo); cf. CIL 2. 2430 (Tarquinus Cathroni); 10. 2009; 8. 24051 (Neronius); 10. 747, 2009 (Neronia);

Tarulius Tatens (CIL 10.687: Surrentum), miles classis; cf. 10. 3600 (Tarullius Rufus), 3387 (C. Tarulius Vitalis) - both Sardinians ?;

Tatia Inc- (CIL 10. 7689: Cagliari): X: 18; VIII: 7; IX: 5; XIV: 2; XI: 3; V: 1;

Tilia (S. 1. 175: Nuragus): X: 30; V: 1; VIII: 1; IX: 3; XI: 2; XIV: 1 ;

Torbenius Kariti (CIL 10. 7876: Fordongianus); cf. 11.6283 (C. Torbanius L. f.);

Urseli Nispenini (dative case ?) (S. 1. 214: Borore); cf. CIL 2. 1385 (Ursili -...), 14. 1679 (Ursella), 1756 (Ursilla);

C. Vehilius C.1. Rufus (CIL 10. 7967: Portotorres): X: 3 ; IX: 1 ; XIV: 2 ; cf. $C I L 10$. 8041. 41, 8046. 27.

Of considerable importance, not only for the nomenclature of Roman Sardinia, but also for the history of migration from the mainland to the provinces in Roman times, is the rather surprising frequency of names which derive from or are most closely paralleled in North Italy, in the region of $C I L 5$ (about $3 \frac{1}{2}$ percent of the total):

C. Acilius Marcianus, Caralitanus (CIL 10.6574: near Vellitri): common in North Italy (V: 23; Alföldy, 54);

M. Alenius Tard- (CIL 10. 7840: Vallermosa): V: 6; IX: 3 ; XI: 8;

C. Asconius C. f. Fab. Sardus (CIL 5. 2829: Patavium); V: 12; XII: 2; XIV: 1;

Atilia L. f. Pomptilla (CIL 10. 7563-78: Cagliari), L. Atilius (10. 7564: Cagliari), L. Atilius Felix (ibid.), Atilius Modestus (CIL 6.3101): most common in North Italy (V: 147), but found elsewhere as well: VIII: 26 ; XI: 66 ; XIV: 28, e.g.;

Benets (S. 1. 177: Ales); if not = Benis, perhaps = Venetus;

Caesilius Hispanus (CIL 10. 7595: Cagliari), Caesilius L. f. Met- (10. 7827: Elmas); Hispanus is fairly common in Spain (Kajanto, 199), but Caesilius is not on record there; it may be North Italian ( $\mathrm{I}^{2}: 1$; III: 1 ; V: 6; VI: 8; VIII: 2 (viz., 8. 2830: Caesilius Hispanus and D. Caesilius D. f. Hispanus); XI: 3; XIV: 4; $A E$, 1940, 92 ; 1959, 141 ;

Calbisius Primitibus (CIL 10. 7636: Cagliari): V: 15; VIII: 12; IX: 3; X: 15; XI: 12; XIV: 3;

Q. Calvisius Euticanus (CIL 10. 7636: Cagliari): Calvisius as above; Euticanus, as a non-Christian name, is especially common in North Italy (Alföldy, 198); 
Q. Catius Firminus (CIL 6. 3105), miles classis: X: 5; V: 43; XI: 17; XIV: 3;

C. Cominius Euplus (CIL 10. 7700: Cagliari): V: 70; X: 26; VIII: 31 ; IX: 18; XIV: 16;

T. Cutius (CIL 10. 7587: Cagliari): II: 4; III: 2; V: 6; VI: 21 ; VIII: 3; X: 2; XI: 3; XII: 1 ; XIV: 9 ;

Enii (CIL 10. 7814: near Pirri), M. Hennius Simphorus (10. 7647: Cagliari): V, IX: 38 each; X: 18; VIII: 12 ; XI: 8 ; XIV: 3 ;

Eusebia Todote (CIL 10. 7630: Cagliari), Eusebius of Vercellae (Bonu, 151-61): common in North Italy (Alföldy, 197; V: 16; IX: 4; XI: 3);

Geminius Ursini (CIL 10. 7657: Cagliari), Geminius Vitalis Primogenitus (ibid.):V: 32; XI: $20 ; \mathrm{X}: 17$; IX: 9 ; VIII: 6 ;

L. Graecinus Felix, Caralitanus (CIL 10. 7891): V: 5; XIV: 2; VIII, IX, X: 1 each (Graecinius);

Grame (CIL 10. 7668: Cagliari): Pape, 259, records Grammis (vocative Grammi), but cf. $C I L$ 5. 1249: Gramme;

A. Licinius Axius (EE 8. 722: Masu); for Axius, II: 1; III: 4; V: 5; VI: 11; VIII: 11; XI: 2;

Mutilia Certa ( $E E$ 8. 713: Cagliari): III: 5; V: 11; VI: 12; VIII: 2; XIV: 3;

C. Oclatius Macer, Caralitanus (CIL 10. 7891): V: 6; VIII: 4; X: 2; XI: 4; XIV: 2 ;

Postumius Srtinianus (CIL 10. 7666: Cagliari): common in North Italy (Alföldy, 112);

C. Quinctius C. f. Quir. F.--tus (CIL 10. 7603: Cagliari): V: 73; IX: 28; X: 25; cf. Alföldy, 114;

C. Rubellius Clytius (CIL 10. 7697: Cagliari): V: 6; VIII: 1; X: 2; XI: 2; XIV: 4;

Secundio (S. 1969, 78: Sulci): V: 8; VIII, X: 5 each;

Sextia Prima (CIL 8. 9198), wife of Abillahas Rummeus V: 34; VIII: 47; IX: 16; $\mathrm{X}: 26$;

Statia Magna P. f. (CIL 10. 7951 : Portotorres): V: 66; VIII: 21 ; IX: 36 ; X: 34; XI: 21 ; cf. Alföldy, 122;

Trofime (S. 1. 344: unknown location): V: 48; X: 29; VIII: 14; cf. Pape, 1558;

Trosius Felix (CIL 10. 7709: Cagliari): very common in V, probably Venetic (Alföldy, 129);

Verona (CIL 10. 7843: Vallermosa).

An unusually high percentage of these names is from Cagliari, with four others from not far away (two at Vallermosa, one each at Pirri and Masu). Only one each is from two other important cities (Portotorres and Sulci), with only one (and that - Benets - a mere possibility) from the interior of the island. A striking fact is beginning to emerge, one which is also clear from the distribution of Greek names on the island (fig. 8), the individual examples of which are too numerous to list here (they comprise about 15 percent of the total).

In contrast stand those names which are either unique to Sardinia (at least as far as I have been able to discover) or which have only one or two parallels elsewhere (about 7 percent of the total):

Acareltorte (S. 1. 217: Ortueri); cf. CIL 10. 8053. 149 (Acar-: Oristano), Hirata, cit.: Acarcelinio;

Aelius Tosuna (S. 1. 153: Pirri); perhaps ef. $C I L$ 11. 2245 (Tosnos: Clusium);

Agbadeus (CIL 10. 7809: Pirri);

Agisinus ( $C I L$ 10. 7855: Tortoli); perhaps cf. $C I L$ 11. 3233 (Agisenna: Nepet);

Arrius Iscini- (CIL 10. 7917 : Cornus); 
Robert J. Rowland, Jr.

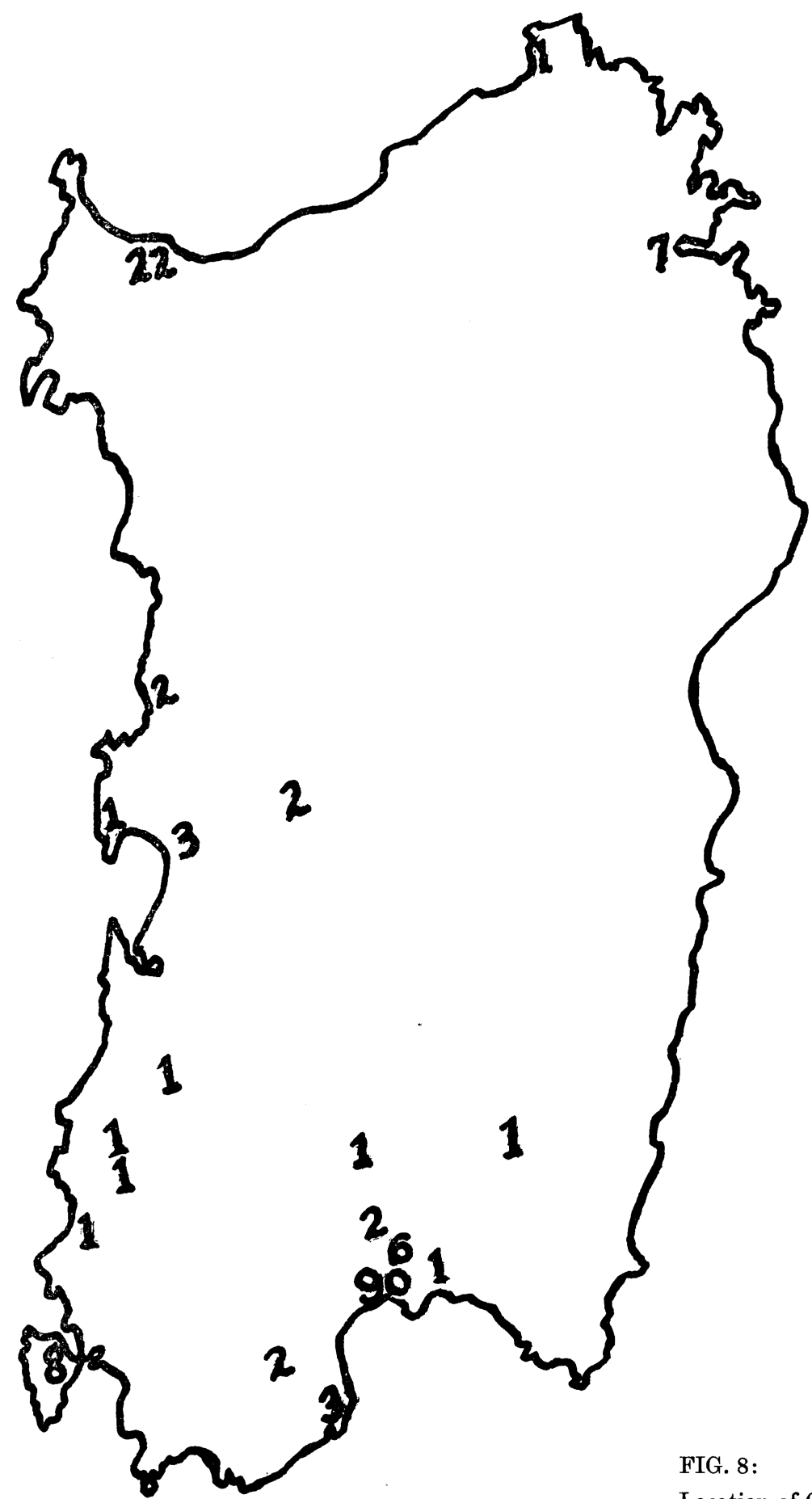

Location of Greek names

$(\mathrm{n}=$ number of examples $)$ 
L. Au-uden- (S. 1. 161: Elmas);

Axtinia Sofia (CIL 10. 7632: Cagliari);

Bacoru (S. 1. 177: Ales); for parallels, see M. Lourdes Albertos Firmat, La onomastica personal primitiva de Hispania Tarraconense y Betica (Salamanca, 1966), p. 47;

Barex (S. 1. 175: Nuragus); perhaps = Baric (VIII: 33), but cf. also CIL 9. 2742: Baris;

Bascio Losonis (filius ?) (CIL 10.7870: Fordongianus); cf. (perhaps) Baskia (Pape, 200);

Batius (= Vatius ?) Rufus ( $E E$ 8. 709: Cagliari), miles classis;

Benets (S. 1. 177: Ales);

C. Beviranus Verus (CIL 10. 7873: Fordongianus);

Bflsa (= Belsa or Beisa) (S. 1. 196: Fordongianus);

Burce (CIL 10. 7809: Pirri); cf. CIL 8. 4897; Pape, 225 (Bourka);

Buttis (CIL 10. 7634: Cagliari); cf. CIL 10.2481 ; 8. 27864; and (perhaps) Pape, 226;

Caritus (S. 1. 196: Fordongianus) and Karitus (CIL 10. 7876: Fordongianus); cf. 8. 1911, 8576;

Cornelius Estutus (CIL 10. 7643: Cagliari);

Curadro (CIL 10. 7854: Ilbone) and Curelo Ner. Cam. (S. 1. 213: Sedilo); cf. Albertos Firmat, cit., p. $101 \mathrm{f}$;

Debos Pat. (S. 1. 176: Nuragus);

Disanirius Torceri (CIL 10. 7872: Fordongianus);

Etunus (CIL 10. 7872: Fordongianus);

Farsonius Occiarius, miles (CIL 10. 7537: Grugua);

Ferentius Miloni f. (S. 1. 224: Ozieri);

C. Fusius Curadronis f. (CIL 10. 7854: Ilbone), miles classis; cf. CIL 2. 6064; 9. 6080. 12, and 11. 6695. 45 (Fusi Scae, indexed as Fusius Scae..);

Gauga Targuronis f. (CIL 10. 7874/5: Fordongianus);

Inivo (S. 1. 180: Laconi);

Ioilus (S. 1. 304: Portotorres);

Iomisus (or Ionisus) (S. 1. 95-6: Cagliari);

Iulia Cranila (= Cornelia ?) (CIL 10. 7644: Cagliari);

Iulia Fortunata quae et Epangielosa (CIL 10. 7670: Cagliari);

Iulia Valeria que et Ginsora ( $E E$ 8. 730: Macomer);

Lucinius Aure. (= Licinius Aurelius?) Nemer N. P. (S. 1. 209: Samugheo); listed as heredes, therefore a plurality;

Lutatia Feifma (?) (CIL 10. 7840: Vallermosa);

Mislius (S. 1. 177 : Ales); perhaps cf. $E E$ 5. 330;

Monioritinus (CIL 10. 7877: Fordongianus);

Nercadaus (CIL 10. 7888: Austis);

Netus (S. 1. 172: Gergei); perhaps cf. CIL 10. 7864; 8. 23250 (Netunus); Holder, 2. 738 (Netis); CIL 12. 5686. 196;

D. Numitorius Agisini f. Tarammo (CIL 10. 7855: Tortoli);

Optatus Sadecis f. ( $A E, 1929,169$ : Mila), Sardus, decurio of a cohors Lusitana;

Otacilia Itageni (S. 1. 269: Portotorres), mother of an Aelius Docimus;

Pertius (EE 8. 737: Telti);

Pilsovicila (CIL 10. 7614: Cagliari);

Pohl (CIL 10. 7650: Cagliari);

Sabdaga (S. 1. 177: Ales); perhaps related to Zabdas;

L. Semmudius (CIL 10. 7882: near Macomer);

Siliso (S. 1. 58: Cagliari);

Silvanus Neti-.. (CIL 10. 7864: Fordongianus);

Socaras Nercanf (?) (S. 1. 232: Tramatza);

Src Unnis (?) (S. 1. 333: unknown location); 
Robert J. Rowland, Jr.

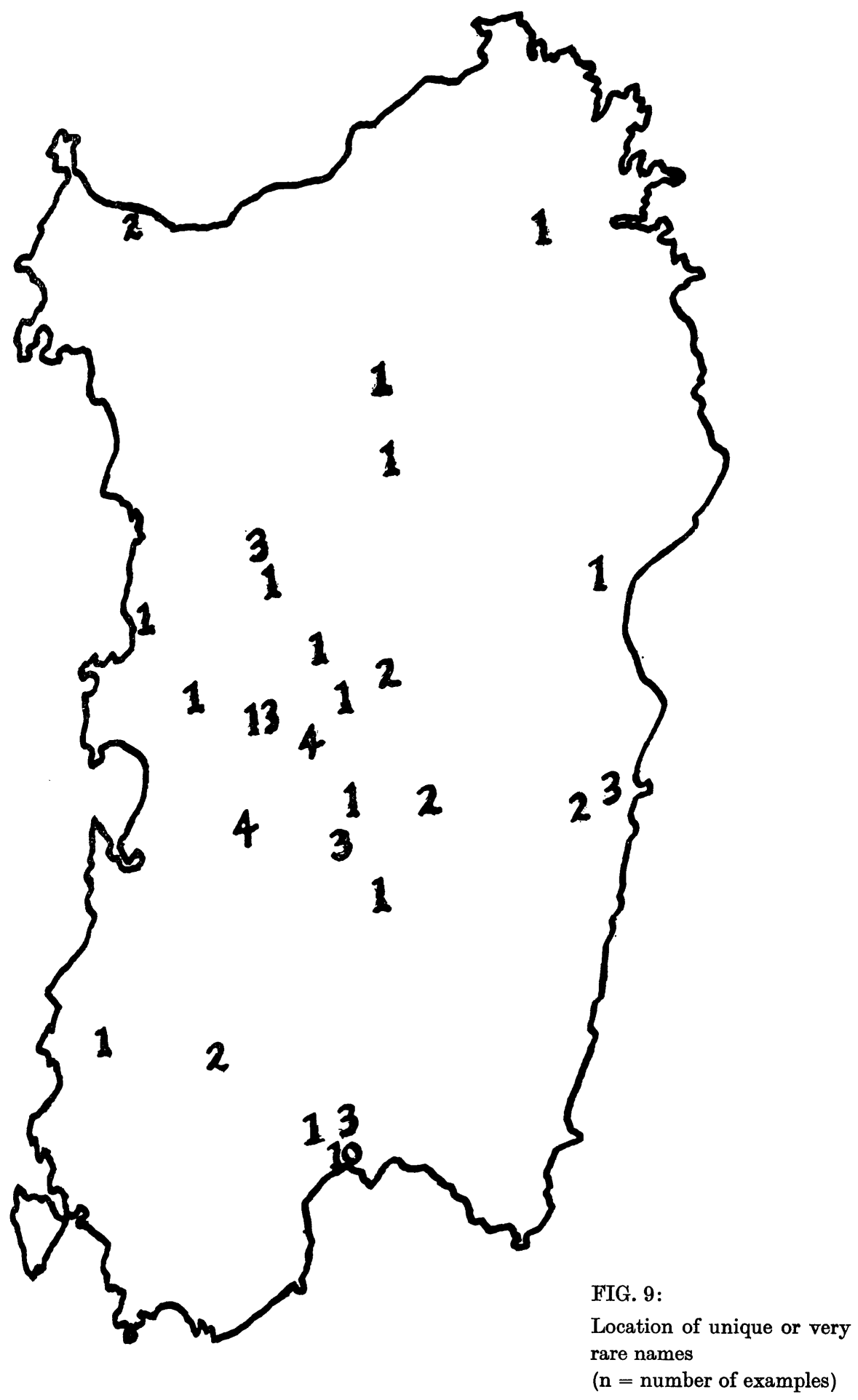


Onomastic Remarks on Roman Sardinia

101

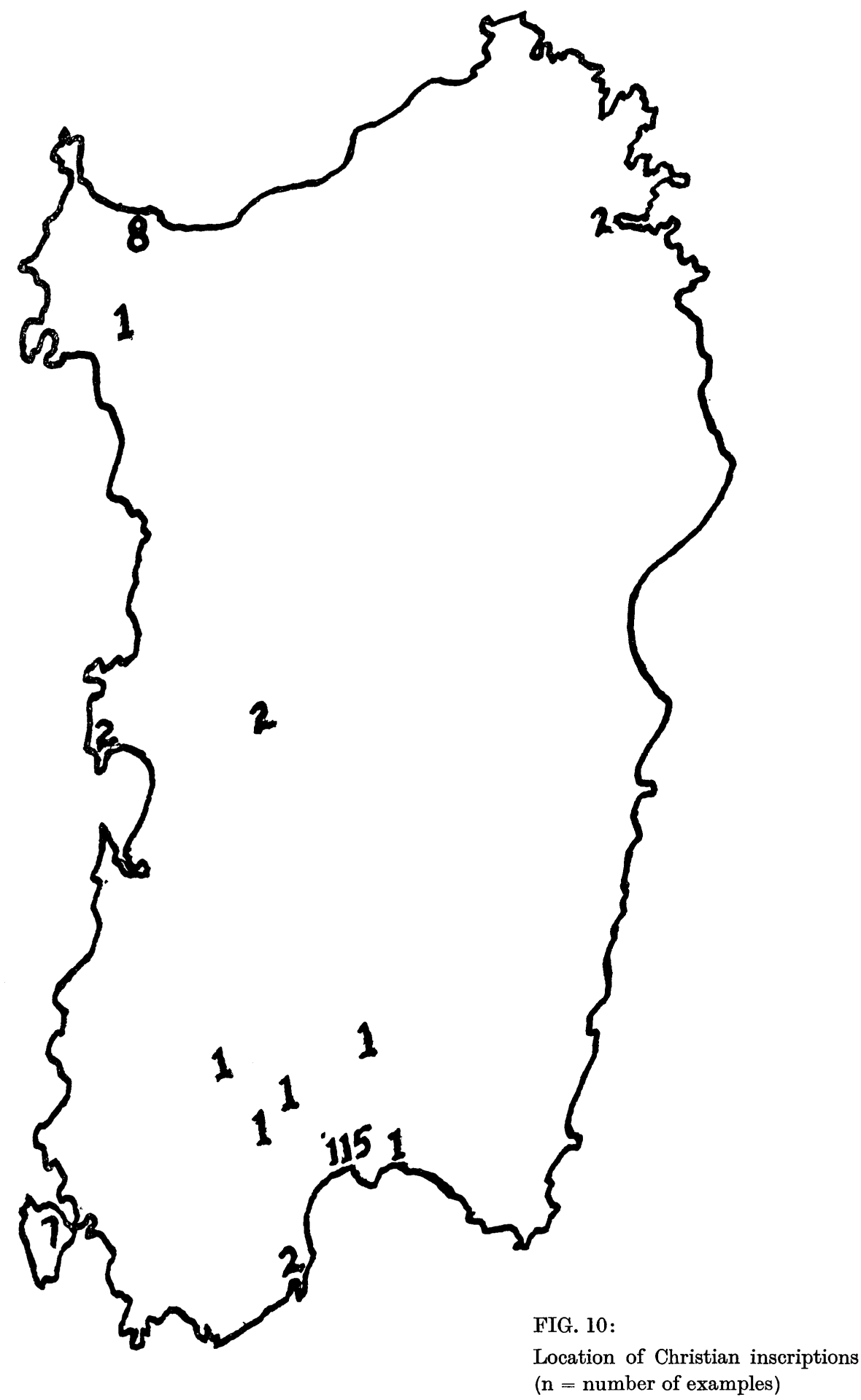


Tarcuinus Fili f. Neroneius (S. 1. 209: Samugheo);

L. Tarcunius Heraclianus ( $A E, 1916,52$ : Rome), miles classis;

C. Tarcutius Tarsaliae fil. Hospitalis (S. 1. 182: Seulo);

Taretius Faric Corneli C. (?) (S. 1. 207: Samugheo);

Targuro (CIL 10. 7874/5: Fordongianus);

Tarpalaris (CIL 10. 7855: Tortoli);

Tarsalia (S. 1. 182: Seulo); cf. CIL 8. 18085a. 16: Tarsa;

Tarulius Tatens (CIL 10.687: Surrentum), miles classis: cf. $C I L$ 10. 3600, 3387;

M. Tigellius (Cic. Fam. 7. 24. lf.; Att. 13.49. lf., 50. 3, 51.2) and M. Tigellius Hermogenes

(Hor. Sat. 1. 2. 1, 3. 1. 9; Ps.-Acro ad 1. 2. 3); cf. CIL 6. 8694;

Torbenius Kariti ( $C I L$ 10. 7876: Fordongianus); cf. $C I L$ 11. 6283;

Tubmar (CIL 10. 7878: Fordongianus);

Tunila Caresio (CIL 10. 7890: Dorgali);

Ubasus Chilonis f. Niclinus (CIL 10. 7884: Austis), tubicen in a cohors Lusitana;

Urrus (S. 1. 174: Nuragus);

Ursaris Tornalis f. (CIL 10.7891: Anela);

Urseli Nispenini (dative case ?) (S. 1. 214: Borore); cf. CIL 2. 1385; 14. 1679, 1756;

Valeria Amoccada (CIL 10. 7842: Vallermosa);

Valeria Urekio ( $E E$ 8. 731 : Macomer);

Valerianus Verseius (S. 1. 208: Samugheo);

Zabarda (Greg. $E p .4$. 25), Dux Sardiniae.

As figure 9 demonstrates, native elements persisted throughout the entire period of the Roman occupation of Sardinia $;^{7}$ and, as is shown both by the series of figures and by the several lists of names, the effects of Romanization - at least in terms of individual nomenclature - are felt largely in the urban, coastal areas, areas which were settled and developed by the Carthaginians and in which Romanization proceeded much as it did in North Africa - even through the Christian period (cf. fig. 10). ${ }^{8}$

\section{University of Missouri}

7 Cf. M. T. Atzori, L'onomastica sarda nei Condaghi, Modena, 1962, 9-11 ("Elemento preromano").

8 I am grateful to the American Philosophical Society for financial assistance and to the Institute for Advanced Study, Princeton for its hospitality in making this study possible. 